\title{
Utilizando o Sistema Integrado de Gestão (ERP) no apoio ao Ensino de Logística e Gestão da Cadeia de Suprimentos
}

\author{
Allan Augusto Platt ${ }^{1}$ \\ Luiz Salgado Klaes ${ }^{2}$
}

\section{Resumo}

O presente trabalho apresenta uma metodologia para o ensino de Logística e Gestão da Cadeia de Suprimentos por meio da utilização de Sistema Integrado de Gestão, também chamado de ERP. Inicialmente define-se o que é Logística, identificando o conjunto de atividades empresariais reunidas sob o seu conceito, $e$ apresentadas aos alunos em sala de aula, bem como o atual conceito de Gestão da Cadeia de Suprimentos, englobando as atividades interorganizacionais. O Sistema Integrado de Gestão (ERP) é caracterizado em seguida, como evolução do Sistema MRP (Material Requirement Planning) e da necessidade das empresas em aperfeiçoarem o controle de suas operações e a tomada de decisões. Exemplos do uso do ERP como ferramenta de apoio no processo de ensino-aprendizagem são demonstrados em seguida. Por último, o trabalho apresenta a possibilidade de utilização do ERP para o ensino dos conteúdos de Logística e Gestão da Cadeia de Suprimentos, tomando como exemplo dois casos desenvolvidos no Brasil.

Palavras-chave: Logística. Gestão da Cadeia de Suprimentos. Sistema Integrado de Gestão. ERP. Ensino.

\section{Introdução}

O ensino de conteúdos da área de Logística e Gestão da Cadeia de Suprimentos tem se pautado, como a maioria dos conteúdos da área de Ad-

\footnotetext{
${ }^{1}$ Doutor pelo Programa de Pós- Graduação em Engenharia de Produção da Universidade Federal de Santa Catarina. Professor da área de Administração de Marketing e Logística do Departamento de Ciências de Administração da Universidade Federal de Santa Catarina. Endereço: Universidade Federal de Santa Catarina, Campus Reitor João David Ferreira Lima, Bairro Trindade - Florianópolis, SC - Brasil - CEP 88.040-970. E-mail: allanplatt14@yahoo.com.br.

${ }^{2}$ Professor da área de Administração de Produção do Departamento de Ciências de Administração da Universidade Federal de Santa Catarina. Endereço: Universidade Federal de Santa Catarina, Campus Reitor João David Ferreira Lima, Bairro Trindade -Florianópolis, SC - Brasil - CEP 88.040-970.E-mail: klaes@cse.ufsc.br.

Artigo recebido em: 25/05/2010. Aceito em: 22/07/2010. Membro do Corpo Editorial Científico responsável pelo processo editorial: Rolando Juan Soliz Estrada.
} 
ministração, nos métodos de ensino-aprendizagem tradicionais. No entanto, com o surgimento de novas ferramentas, fruto do grande avanço da tecnologia da informação, como sistemas de informação avançados, softwares de simulação, uso da Internet para disponibilização de conteúdos e interação alunoprofessor, incluindo as tecnologias de ensino a distância, tem ocorrido investidas acadêmicas em busca de novas alternativas de ensino.

Uma das tecnologias mais utilizadas nos últimos anos, enfatizada pelo interesse de seus fornecedores em divulgar e disseminar em universidades, sobretudo, em faculdades de Administração, Engenharia e Computação, é o Sistema Integrado de Gestão, ou como é popularmente conhecido, o ERP.

$\mathrm{O}$ uso do ERP no meio acadêmico passa desde a simples navegação em seus módulos, ilustrando conteúdos nas áreas de Sistemas de Informação, até a própria simulação de operações (transações) em suas várias funcionalidades, contextualizando o que é visto pelos alunos em sala de aula, em conteúdos das disciplinas de Finanças, Recursos Humanos, Marketing, Produção e Logística, entre outros, ilustrando a visão empresarial atual de gerenciamento de processos, em contrapartida à visão tradicional funcional e departamental, como no desenvolvimento de habilidades junto a um Sistema de Informação.

\section{Logística e Gestão da Cadeia de Suprimentos}

A logística tem origem militar, sendo descrita por Heródoto como parte das artes militares que se destina a assegurar às forças armadas todos os meios necessários para a sua sobrevivência no campo de batalha, incluindo melhores condições de movimentação, abastecimento, alojamento e transporte de tropas (MEDINA, 2002). Voltou a ser ressaltada no período pós-guerra, em que as atividades logísticas militares utilizadas na II Guerra Mundial influenciaram significativamente os conceitos logísticos utilizados atualmente.

Um desses conceitos é apresentado por Ballou (2001, p. 21):

[...] é o processo de planejamento, implementação e controle do fluxo eficiente e economicamente eficaz de matérias-primas, estoque em processo, produtos acabados $e$ informações relativas desde o ponto de origem até o ponto de consumo, com o propósito de atender às exigências dos clientes. 
Já Christopher (1997, p. 2) define logística como:

[...] o processo de gerenciar estrategicamente a aquisição, movimentação e armazenagem de materiais, peças e produtos acabados (e os fluxos de informações correlatos) através da organização e seus canais de marketing, de modo a poder maximizar as lucratividades presente e futura por meio de atendimento dos pedidos a baixo custo.

O conceito de logística, de acordo com os autores, relaciona-se com o gerenciamento e execução das atividades ao longo de toda a cadeia de suprimentos, incluindo atividades junto a fornecedores e clientes, visando a racionalizar os fluxos físicos e informacionais e à satisfação das demandas dos consumidores. A Figura 1 ilustra esquematicamente esses fluxos:

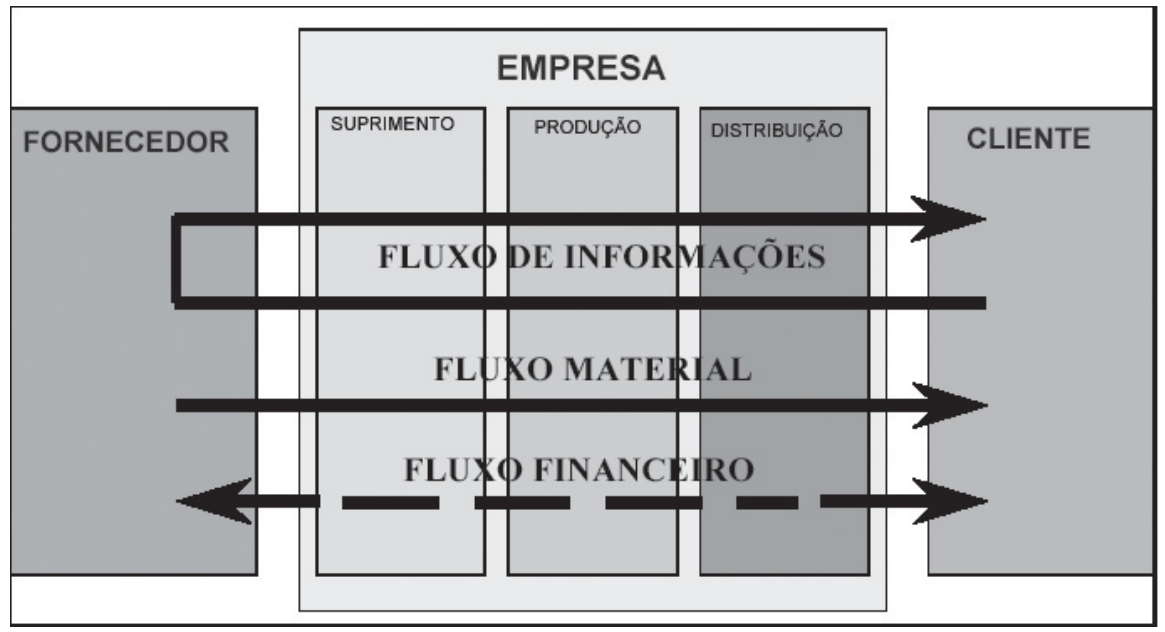

Figura 1: Fluxos logísticos

Fonte: Elaborada pelos autores

Para Medina (2002), a logística, hoje, é considerada uma abordagem gerencial, que trata de forma sistêmica e integrada todas as atividades relacionadas aos fluxos físicos, financeiros e de informações da organização. No início, suas atividades eram gerenciadas de forma isolada, atreladas a outros departamentos da empresa (finanças e marketing, por exemplo), até os dias de hoje, com uma abordagem integrada de suas funções, como ilustra a Figura 2: 


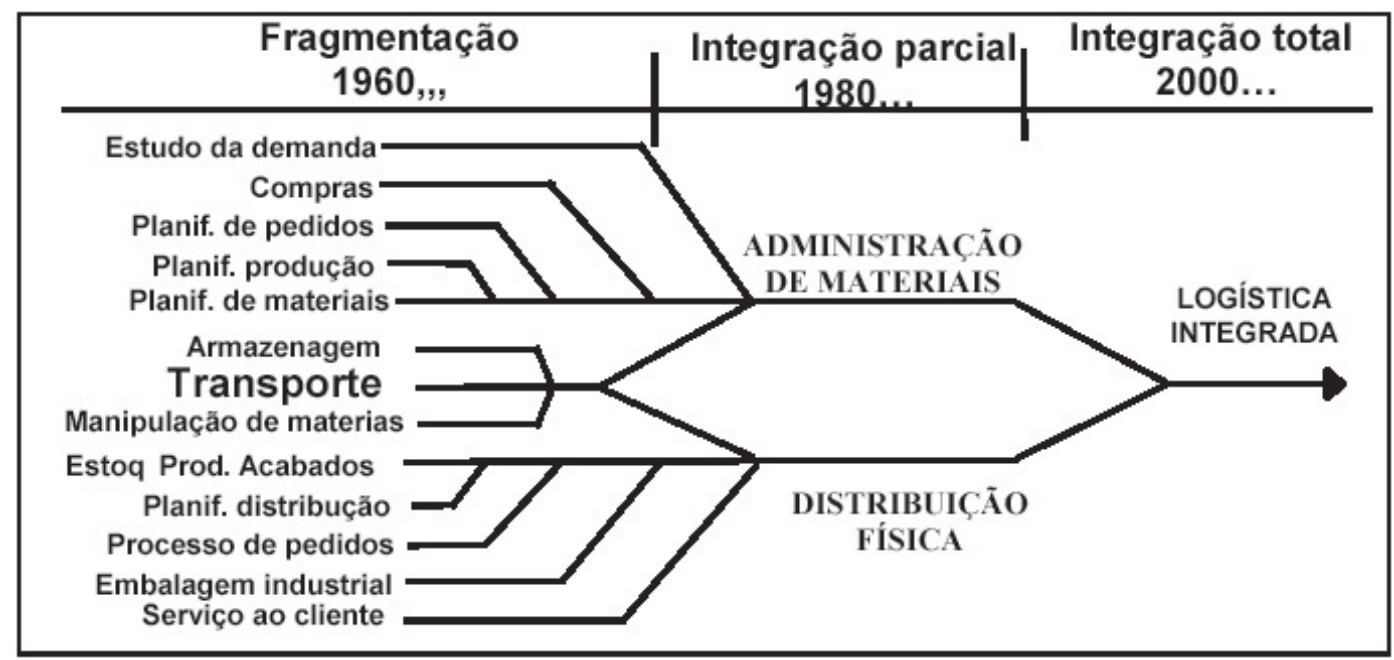

Figura 2: Evolução logística a partir de 1960

Fonte; Elaborada pelos autores

Com a evolução natural do conceito de logística integrada, representando uma integração interna de atividades, surge o conceito de Supply Chain Management (Gestão da Cadeia de Suprimentos) que traduz a integração externa das atividades da empresa em uma série de processos interligando seus fornecedores, clientes e consumidores finais.

Conjugando os processos logísticos, que tratam do fluxo de materiais e informações dentro e fora das empresas, com os relacionamentos que surgem ao longo dessa cadeia (Figura 3), a Gestão integrada da Cadeia de Suprimentos visa a assegurar resultados às empresas tanto em termos de redução de desperdício como em agregação de valor. Questões colaborativas definidas por esse conceito integrativo, como a formação de parcerias e de alianças estratégicas logísticas, visam a explorar as atividades logísticas em busca de vantagens mútuas (FIGUEIREDO; ARKADER, 1998). 


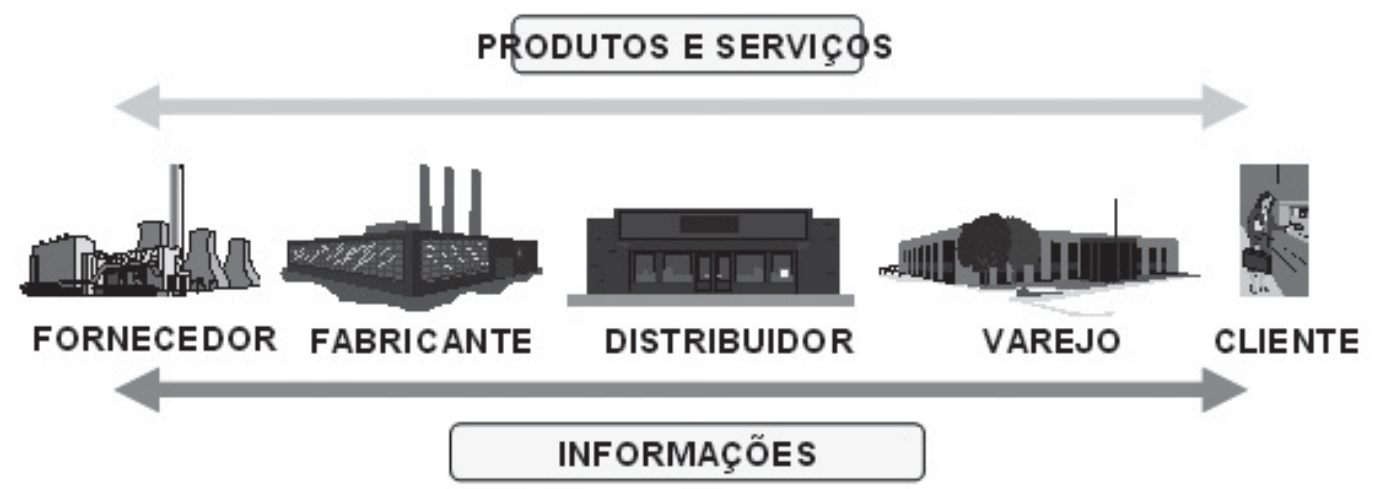

Figura 3: Esquema ilustrativo de uma cadeia de suprimentos

Fonte: Elaborada pelos autores

Em relação ao ensino dessa área, Figueiredo e Arkader (1998) comentam que em escolas líderes no ensino de logística, como a da universidade de Michigan (MSU), foram realizadas fusões entre professores e pesquisadores das áreas de Operações (incluindo Logística e Produção) e Marketing, criando uma área multidisciplinar de Gestão da Cadeia de Suprimentos. Quanto aos conteúdos, destacam-se o uso de sistemas de informação e outras inovações proporcionadas pelo avanço nas tecnologias da informação, como EDI que facilitam a integração dos elos da cadeia, bem como a disseminação de conceitos gerenciais como o JIT (Just in Time), QR (Quick Response) e o ECR (Efficient Consumer Response), além do uso das tecnologias de informação para simulações de situações com as quais os alunos irão se deparar no mercado.

\section{Sistema Integrado de Gestão (ERP)}

O avanço da tecnologia de informação permitiu que as organizações incorporassem sistemas informatizados no apoio às suas atividades. Um dos expoentes desse avanço são os sistemas de informação empresariais, desenvolvidos para atender aos requisitos específicos das diversas áreas e departamentos.

Dávalos e López (2002) apresentam os sistemas de informação como um conjunto de componentes inter-relacionados, desenvolvidos para coletar, processar, armazenar e distribuir informações, facilitando a coordenação, o controle, a análise, a visualização e o processo decisório nas organizações. 
No entanto, esses sistemas de informação foram desenvolvidos como rebatimento às estruturas organizacionais funcionais e departamentais. Surgem assim, sistemas que suportam as necessidades financeiras da empresa, outros voltados à produção, compras, outros, ainda, para o gerenciamento de estoques, contabilidade e recursos humanos. Como consequência, verifica-se que a operacionalização, controle e tomada de decisões nas empresas é sustentada por uma grande quantidade de sistemas, com linguagens de programação e bases de dados distintas, dificultando o processo de gestão.

Adicionado à pressão competitiva global em busca das melhores práticas, a promoção da reengenharia de processos de negócio pelas grandes firmas de consultoria, a tendência internacional de privatização dos serviços públicos, a ampliação dos recursos destinados à área de Tecnologia da Informação para suportar a reengenharia das empresas e a pressão para mudanças rápidas de adequação dos sistemas de informação às necessidades da empresa, surgem os fatores necessários para o desenvolvimento dos sistemas integrados de gestão, também denominados de ERPs (WIEDER, 1999).

O termo ERP (Enterprise Resource Planning), deriva de um trocadilho para os sistemas que o antecederam, denominados MRP ((Material Requirement Planning) e MRP II (Manufacturing Resource Planning). Esses sistemas estão relacionados ao apoio às áreas de produção e suprimentos da empresa que evoluíram, abrangendo funções contábeis e financeiras e, por último, todas as principais funções gerenciais da organização.

A estrutura de um sistema integrado de gestão é composta de módulos que incorporam os processos de negócio da organização, baseados nas melhores práticas mundiais de execução desses processos e desenvolvidas por fornecedores como SAP, Oracle, JDEdwards, Peoplesoft, Migrosiga e Datasul.

Suas características principais são:

a) orientação à gestão por processos ao invés de gerenciamento funcional e departamental, característica dos sistemas legados;

b) multifuncionais, incorporando processos de compras, vendas, finanças, entre outros;

c) possuem uma base de dados única, permitindo a integração dos processos;

d) modular, possibilitando que sejam utilizados com qualquer combinação de módulos; 
e) estruturados sob a arquitetura cliente/servidor; $e$

f) expansíveis, permitindo a integração externa com interfaces com empresas parceiras e a utilização do comércio eletrônico (PLATT, 2004).

A Figura 4 ilustra a estrutura de um ERP:

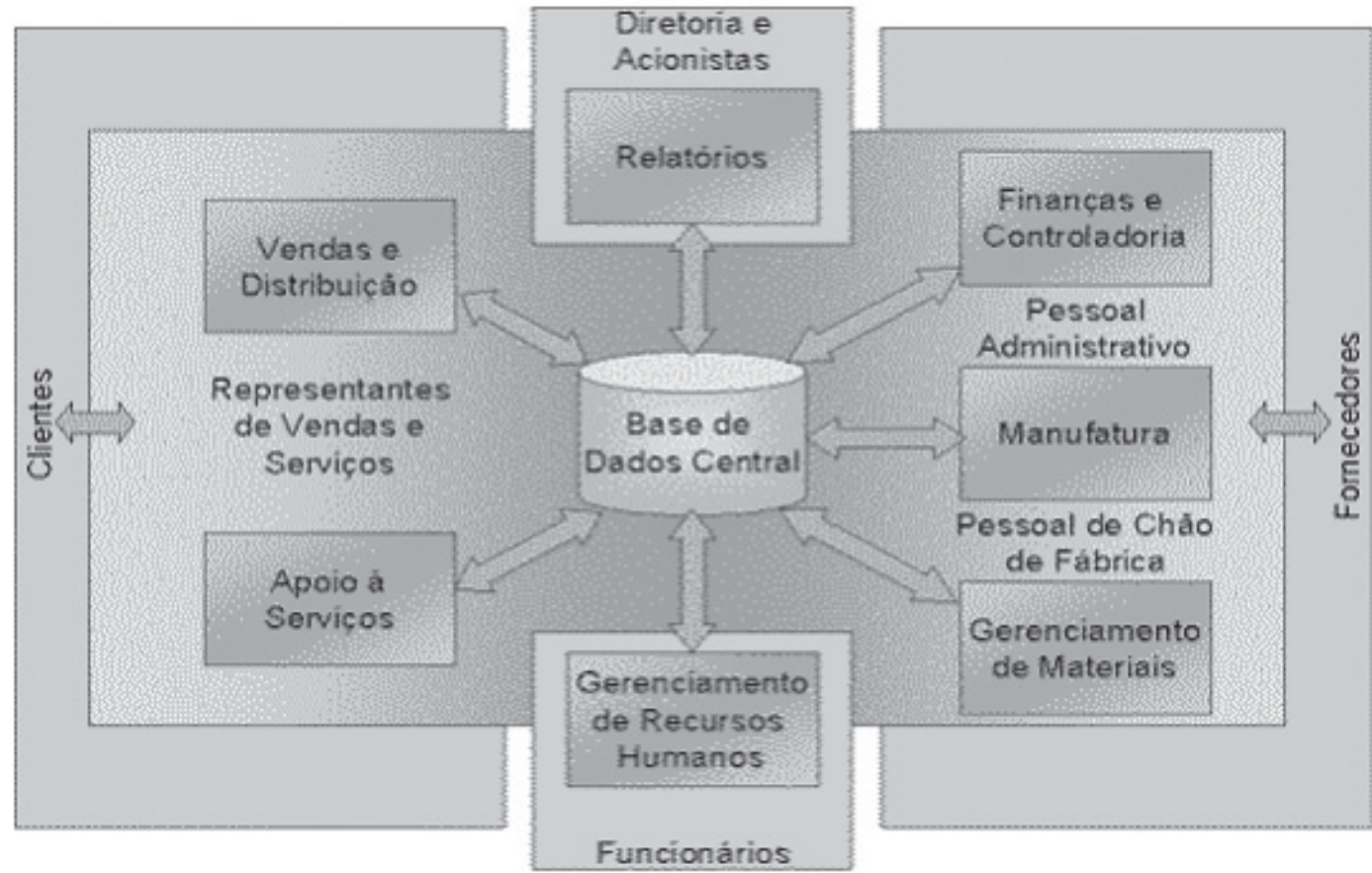

Figura 4: Estrutura típica de funcionamento de um sistema ERP

Fonte: Elaborada pelos autores

\section{O Sistema ERP no Ensino Superior}

Inúmeras parcerias foram desenvolvidas entre universidades e empresas fornecedoras de Sistemas ERP nos últimos dez anos, como apresentam Watson e Schneider (1999), Wieder (1999), Becerra-Fernandez e Murphy (2000), Guthrie e Guthrie (2000), Antonucci e Meuhlen (2001), Kurihara e Breternitz (2001), Hawking e McCarthy (2001), Hawking et al. (2002), Dávalos e López (2002), Dávalos e Platt (2002), Platt et al. (2004). 
Para alguns desses autores, há uma clara oportunidade para as universidades obterem valor agregado e percebido em seus currículos dos cursos voltados aos negócios, engenharia e computação, aumentando a empregabilidade dos recém-formados e elevando a procura por matrículas nestes cursos (WATSON; SCHNEIDER, 1999; GUTHRIE; GUTHRIE, 2000).

Em contrapartida, a formação de profissionais graduados com habilidades nestas ferramentas (ERP), garantindo propaganda e custos mais baixos de implementação de seus sistemas, além de associação de seus nomes às instituições, são fatores que impulsionam os fornecedores de ERP na realização dessas parcerias (HAWKING; MCCARTHY, 2001).

Para Guthrie, Guthrie (2000), como o ERP é um modelo empresarial baseado em computador, ao ser trazido para o ambiente pedagógico, poderá ser uma ferramenta muito útil, demonstrando a relevância do conteúdo do curso para o mundo real, além de permitir o aprendizado prático junto ao sistema, através da navegação em seus módulos, na execução de atividades (transações) e na produção de relatórios gerenciais.

No entanto, um plano que determine tempo, esforço do pessoal (tanto da instituição como do fornecedor do ERP) e recursos para infraestrutura, deve ser elaborado para que a parceria tenha sucesso. Watson e Schneider (1999) colocam que esse plano deve estar sincronizado com os objetivos da parceria que geralmente são identificados desta forma:
a) desenvolvimento de um simulador computacional;
b) exposição dos alunos às situações do mundo real;
c) desenvolvimento de um currículo interdisciplinar;
d) enriquecimento de currículos específicos com o uso do sistema ERP;
e) desenvolvimento de oportunidades de novas pesquisas; $e$
f) criação de vantagem competitiva. 


\section{O ERP no Ensino de Logística e Gestão da Cadeia de Suprimentos}

Como um sistema que vem configurado com as melhores práticas de negócio, o ERP permite o registro, processamento e armazenamento de dados relativos às atividades empresariais, tornando-se uma ferramenta que pode auxiliar o processo de ensino-aprendizagem, sobretudo, das Ciências da Administração, tanto na contextualização de conteúdos das disciplinas do curso, como na visão de processos integrados, além do próprio funcionamento da prática empresarial e de um sistema de informações que o represente e auxilie o processo de tomada de decisões.

O ensino da Logística Empresarial pode se beneficiar do uso do ERP na operacionalização de conteúdos práticos relacionados à gestão de Estoques (análise $\mathrm{ABC}$, elaboração de previsões e tendências de consumo, configurações de MRP, com estabelecimento no sistema de ponto de pedido, lote de compra, estoque de segurança, determinação dos custos de estoque, entre outros), gestão de compras (elaboração de todo o processo, desde as requisições, verificação em estoque, elaboração de cotações, ordenação no sistema das alternativas cotadas, elaboração de pedido, etc.), bem como de suas atividades operacionais de armazenagem, movimentação, classificação, transportes de materiais, entre outras, através da realização de atividades e processos no próprio módulo do sistema.

No entanto, com o avanço do conceito de Gestão da Cadeia de Suprimentos, os conteúdos de Logística se ampliam para fora das fronteiras da empresa, exigindo contextualização de conceitos de integração não apenas interfuncional como interorganizacional, através das transações integradas. Um exemplo clássico é o da realização de um processo de gestão do pedido, com a elaboração de atividades pré-venda, realização do pedido de um cliente via sistema, análise de estoque para verificar disponibilização dos itens, montagem do pedido, definição do roteiro de entrega, faturamento e contas a receber como ilustra a Figura 5: 


\section{Customer Order Management}

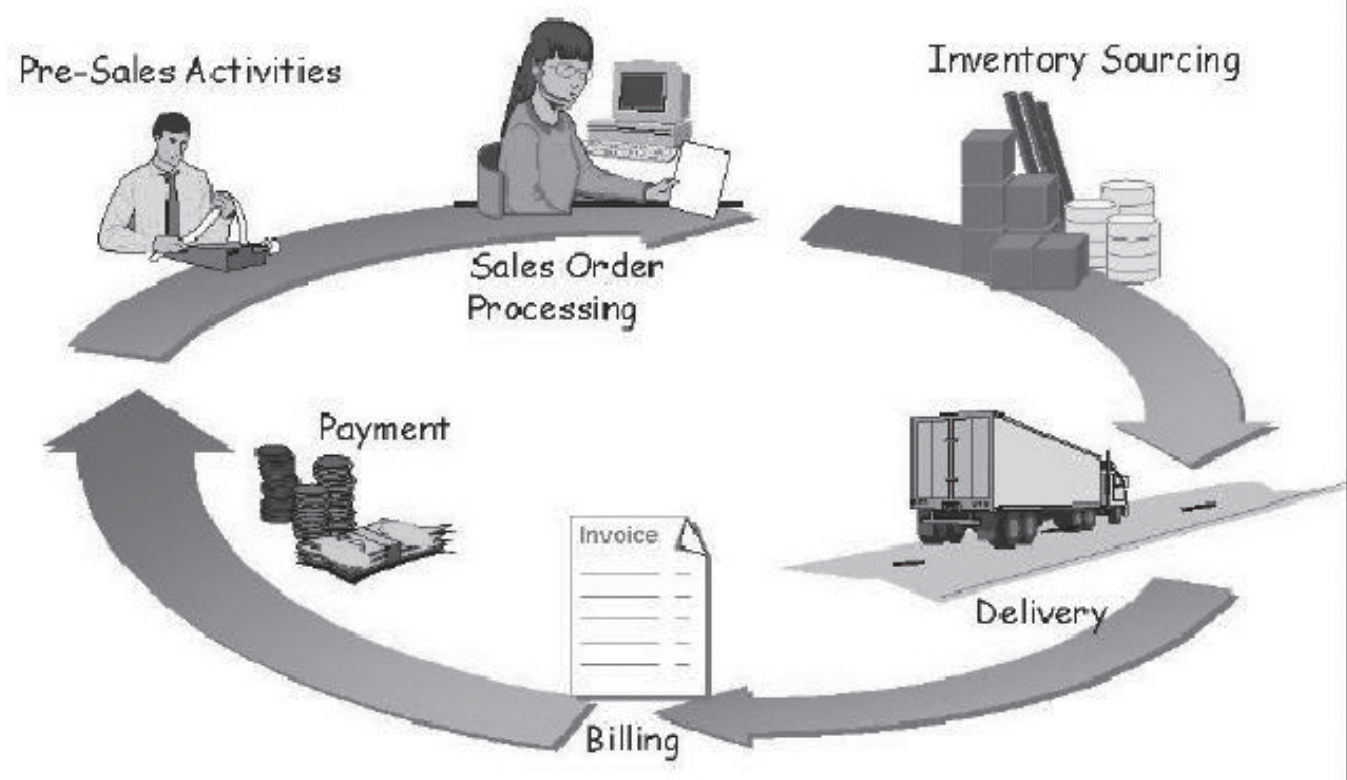

Figura 5: Processo de negócio de gestão de pedido

Fonte: Elaborada pelos autores

Caso não haja disponibilidade em estoque, é possível após a configuração do MRP, enviar uma ordem de produção, realizar pedidos aos fornecedores via comércio eletrônico, registrar o recebimento das matérias-primas, incorporar os itens em estoque, programar a produção, determinar os custos dos produtos, realizar o pagamento das matérias-primas, enfim, realizar, através de simulação, todas as atividades de uma Cadeia de Suprimentos tradicional.

Além dessas atividades, o conhecimento desses conteúdos junto ao ERP é aprofundado com a parametrização do sistema pelos alunos, por meio da criação de clientes, fornecedores, produtos, armazéns e, principalmente, de relatórios gerenciais, desenvolvendo nos alunos habilidades gerenciais exigidas no exercício de sua futura profissão.

É importante ressaltar a visão de processos do sistema que irá propiciar aos alunos e professores uma visão integrada de empresa e, 
consequentemente, de curso, com boas possibilidades de integração com disciplinas de Contabilidade e Custos, Administração Financeira, Economia, Administração de Marketing, Administração de Produção, Importação e Exportação, Administração de Sistemas de Informação, Comércio Eletrônico, entre outras.

Como sistemáticas para uso do ERP, Guthrie e Guthrie (2000) apresentam três possibilidades de integração com currículos universitários: intermodular, modular do currículo e interdisciplinar.

A primeira abordagem possibilita uma visão do sistema como um todo, com exposição geral de todos os módulos, podendo ser realizada por uma disciplina como Sistema de Informações e anterior a de áreas-fim de uma empresa, como Logística e Gestão da Cadeia de Suprimentos, Finanças, Marketing, etc.

$\mathrm{Na}$ integração modular do currículo, os alunos cursam uma disciplina e aprendem os conceitos no módulo do ERP correspondente. Aqui, os alunos podem aprender os conceitos logísticos de gestão de estoques e compras configurando o sistema e realizando transações no sistema que contextualizem o que aprendem em sala de aula.

Já com a integração interdisciplinar, o conceito de processos integrados é colocado à prova, com a realização de processos envolvendo conceitos de várias disciplinas, como o ilustrado anteriormente de ciclo de pedido (Figura 5).

Atividades mais complexas como a de Implementação de um módulo de ERP, em que é possível customizar um processo, adequando-o à realidade de uma empresa específica, determinada pelo professor da disciplina e a Simulação, em que é permitido ao aluno comparar as melhores práticas de negócio, incorporadas no sistema e desenvolver alternativas possíveis de processos específicos da empresa, incluindo análise de gaps são citadas por Watson e Schneider (1999) como alternativas mais avançadas no processo de ensino-aprendizagem.

Exemplos brasileiros de utilização de conceitos de Logística e Gestão da Cadeia de Suprimentos no meio acadêmico, utilizando o ERP, são escassos ainda. O trabalho mais relevante foi desenvolvido pelo Núcleo de Manufatura Avançada (NUMA) da USP - São Carlos, eleito o melhor projeto das Américas no "SAP Research and Development Grant Award", que apoia as melhores propostas de utilização do Sistema R/3 (ERP da SAP) em iniciativas educacionais e de pesquisa. 
Denominado IPROS ("Integrated Production and Supply Chain Management"), o projeto desenvolveu um simulador de cockpit de produção, baseado nas aplicações do $\mathrm{R} / 3$ para o gerenciamento da Cadeia de $\mathrm{Su}$ primentos. Baseado em conceitos de integração interorganizacional, o projeto permite, por exemplo, que os dados de uma máquina que quebrou e a informação de que algum segmento da produção está atrasado pode ser útil e interessar não apenas quem está dentro da empresa, como também a outros elos da cadeia produtiva, como um fornecedor de matéria-prima. Essas informações ainda podem gerar novos indicadores e auxiliar no desenvolvimento de novos padrões na inteligência de negócios, bem como no replanejamento e na tomada de decisões dentro da fábrica. O projeto IPROS apresenta o cenário conceitual de uma Cadeia de Suprimentos do setor metal-mecânico como representado na Figura 6, com posterior construção de um cockpit de produção.

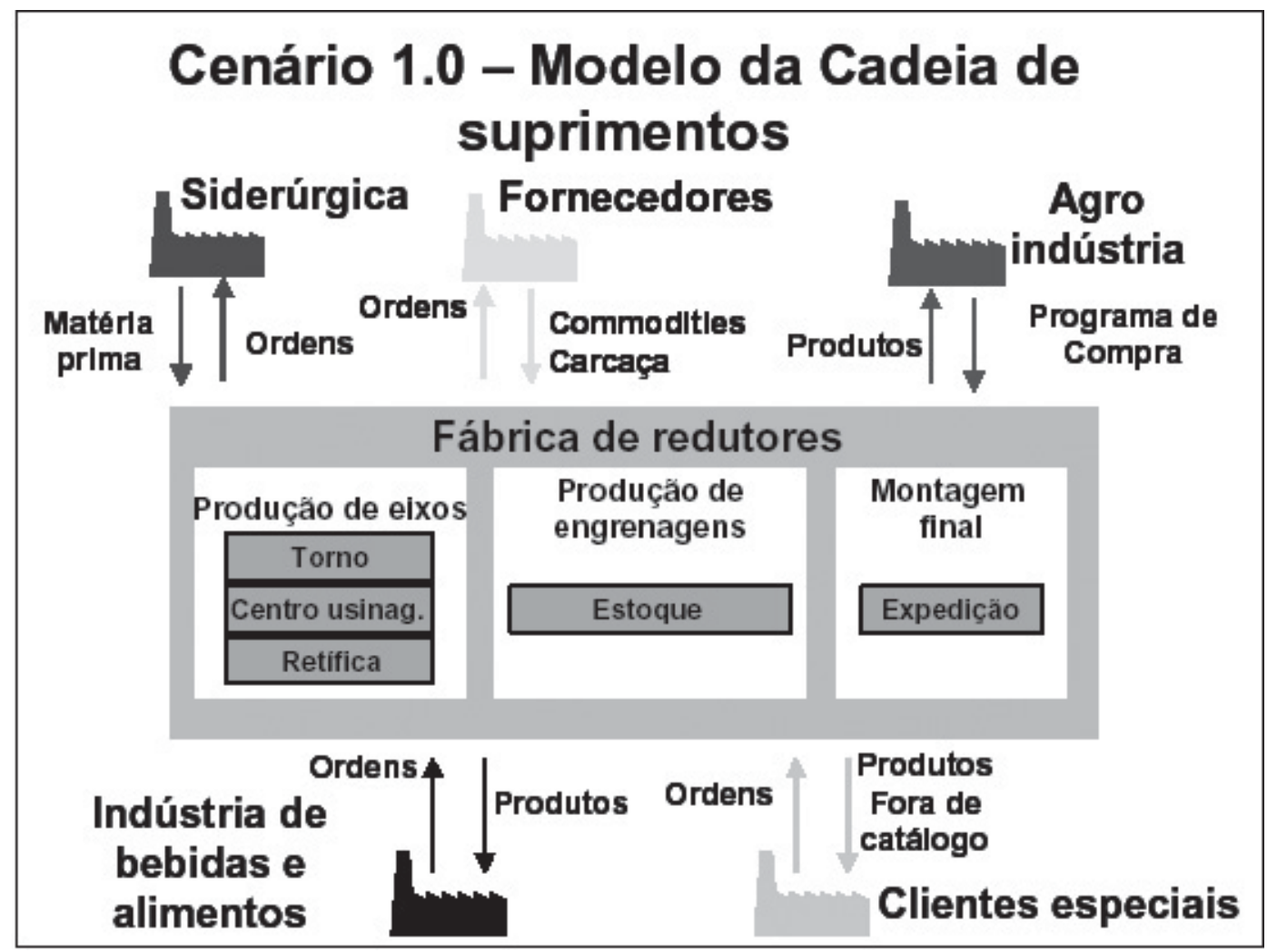

Figura 6: Modelo da cadeia de suprimentos do projeto IPROS Fonte: Elaborada pelos autores 
Platt (2004) apresenta um trabalho realizado nessa área por um grupo de pesquisadores da Universidade do Sul de Santa Catarina (UNISUL) que criou um modelo que simula uma Cadeia de Suprimentos, configurando o ERP de acordo com as características econômicas e os cursos oferecidos em cada Campus da respectiva Universidade (Figura 7).

Os alunos dos cursos de negócio (Administração, Contabilidade, Economia) dos quatro Campus são agrupados em quatro empresas representadas na Cadeia de Suprimentos. Nessas empresas, eles desenvolvem atividades empresarias relacionadas à compras, finanças, produção, vendas e recursos humanos, além das interações comerciais do tipo B2B (comércio eletrônico entre empresas) e de Supply Chain Management (Gestão da Cadeia de Suprimentos).

Já os alunos das áreas tecnológicas (Computação, Sistemas de Informação e Engenharias) são reunidos em dois grupos, constituindo duas empresas de suporte para as quatro empresas da Cadeia de Suprimentos, as quais desenvolverão atividades de aprimoramento, administração dos sistemas e de suas bases de dados, desenvolvimento de customizações, etc.

A estratégia de alocação das empresas fictícias em função dos Campus atende aos interesses da UNISUL em contextualizar seus conteúdos com a vocação da região onde o respectivo Campus está instalado. O critério relacionado aos cursos oferecidos nos Campus também é levado em conta para a localização das empresas da seguinte forma:

a) Empresa fornecedora (instalada no laboratório do Campus Araranguá) é responsável pelas atividades de beneficiamento de matérias-primas.

b) Empresa industrial (instalada no laboratório Campus Tubarão) é a responsável pelas atividades de compra e montagem de produto.

c) Empresa varejista 1 (instalada no laboratório do Campus da Grande Florianópolis) e empresa varejista 2 (instalada no laboratório do Campus do Norte da Ilha) responsabilizam-se pela compra e venda de produtos ao consumidor final.

d) Empresa fornecedora de serviços de suporte 1 (instalada no laboratório do Campus Tubarão) atua no desenvolvimento de aplicativos, customizações e administração da base de dados e dos sistemas das empresas fornecedora (Campus Araranguá) e da produtora (Campus Tubarão). 
e) Empresa fornecedora de serviços de suporte 2 (instalada no laboratório do Campus da Grande Florianópolis) atua no desenvolvimento de aplicativos, customizações e administração da base de dados e dos sistemas das empresas varejistas.

Fluxo de suporte

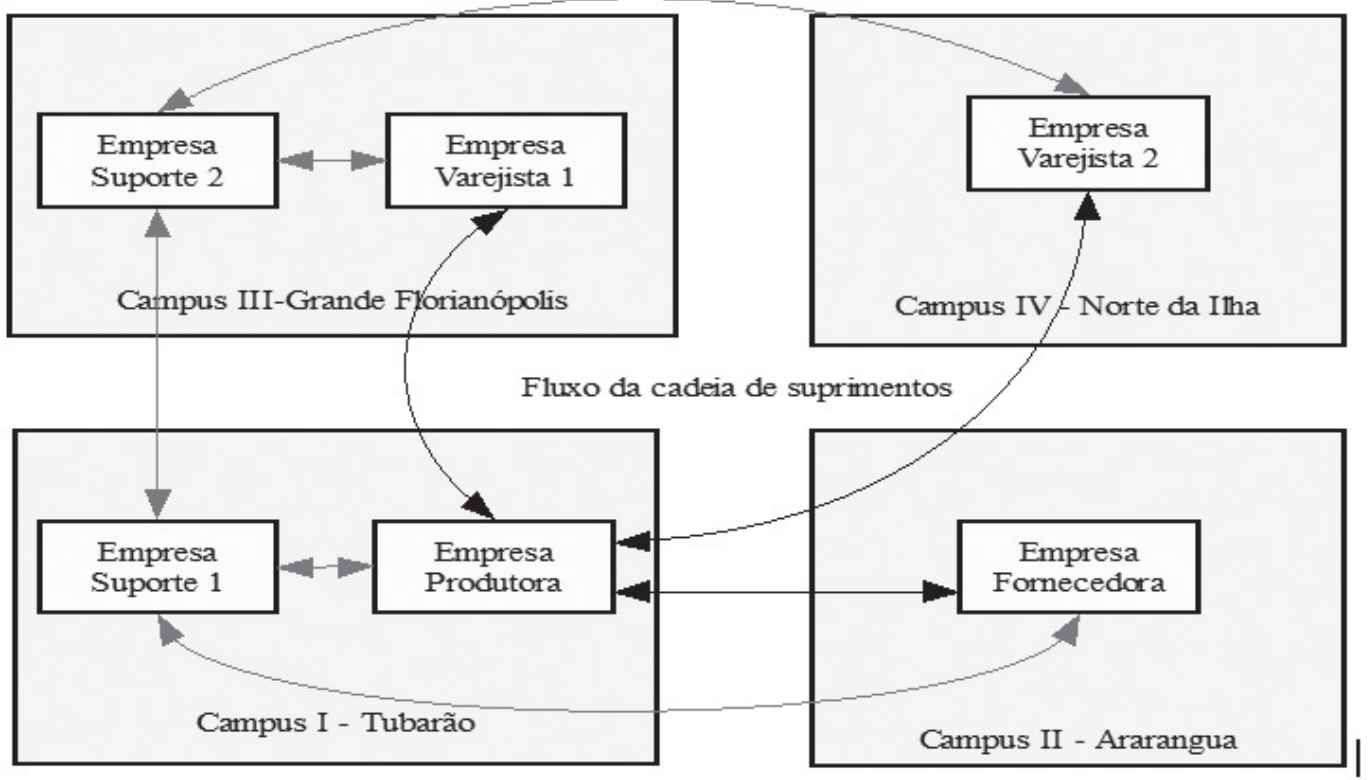

Figura 7: Modelo abrangente que inclui o sistema ERP na UNISUL Fonte: Elaborada pelos autores

Encerrando, é possível verificar que tanto na contextualização de conteúdos como no desenvolvimento de simulações, vislumbram-se grandes alternativas ao ensino de Logística e Gestão da Cadeia de Suprimentos através do uso de sistemas integrados de gestão.

Os exemplos de interação aluno-sistema, através da realização de transações nos módulos do ERP, em processos integrados com outras áreas, possibilitarão, além de mais conhecimentos sobre um sistema integrado de gestão e processos logísticos de negócio, o desenvolvimento de habilidades gerenciais fundamentais ao exercício da profissão de Administrador. 


\section{Considerações Finais}

Este trabalho apresentou alternativas de uso de sistema integrado de gestão como ferramenta de apoio no processo de ensino-aprendizagem de Logística e Gestão da Cadeia de Suprimentos.

A possibilidade de utilização de uma ferramenta ERP como recurso de apoio ao processo de ensino aprendizagem é muito valiosa, pois permite, por meio de simulação, que o aluno tenha acesso à interação prática com um sistema que representa os processos de negócio de uma empresa. A contextualização dos conteúdos de Logística e Gestão da Cadeia de Suprimentos com a realização de transações no sistema, de processos integrados com outras áreas da organização, análises $\mathrm{ABC}$, realização de previsões, configuração de MRP, determinando ponto de pedido, estoque de segurança, custos de estoque, processamento de pedidos, comércio eletrônico, entre outros podem ser estudados com base no ERP.

Através de dois casos apresentados, é possível verificar que além do uso para transações empresariais, o uso do ERP também permite extrapolar o aprendizado para todas as interações em uma Cadeia de Suprimentos, trazendo aos alunos uma visão integrada de gerenciamento e possibilitando análises mais profundas para a tomada de decisão.

O grande desafio que surge a partir de uma iniciativa desse tipo é o delineamento de um projeto que permita a união de instituição, por meio de seus cursos, coordenadores, professores, técnicos e alunos e pelo fornecedor do sistema, com seu software e equipe de consultores, para iniciarem uma parceria que, entre inúmeros benefícios, irá formar pessoal mais qualificado e sintonizado com a realidade do mercado. 


\section{Utilizing the Enterprise Resource Plannig (ERP) Supporting Logistcs Teaches and Suppli Chain Management}

\section{Abstract}

The present work introduces one methodology for Logistics and Supply Chain Management teaching through an utilization of an Enterprise Resource Planning, called also ERP. In the first place it is defined what Logistics is, identifying the conjunct of business activities grouped under your concept, and presented to the students in the class, as well the actual concept of Supply Chain Management, covering the inter organization activities. The Enterprise Resource Planning (ERP) is afterwards characterized, like an evolution of MRP System (Material Requirement Planning) and the necessity of companies improve the control of yours operations and decision-making. Examples about the use of ERP like a support instrument in the process of teaching/ learning are demonstrated right away. At last the work present the possibility of ERP utilization for teaching subjects like Logistics and Supply Chain Management, clarifying using two cases developed in Brazil.

Key words: Logistics. Supply Chain Management. Enterprise Resource Planning. ERP. Teaching.

\section{Referências}

ANTONUCCI, Y. L.; MEUHLEN, M. Zur. "Deployment of Business to Business Scenarios in ERP Education: Evaluation and Experiences from an International Collaboration," Proceedings of the Seventh Americas Conference on Information Systems, Miami, p. 998-1004, 2001.

BALLOU, Ronald $\mathrm{H}$. Gerenciamento da Cadeia de suprimentos: planejamento, organização e logística empresarial. 4. ed. Porto Alegre: Bookman, 2001.

BECERRA-FERNANDEZ, I.; MURPHY, K. E. "Integrating ERP in the business school curriculum". Communications of the ACM, v. 43, n. 4, 2000.

CHRISTOPHER, M. Logística e Gerenciamento da Cadeia de Suprimentos: estratégias para a redução de custos e melhoria dos serviços. São Paulo: Pioneira, 1997. 
DÁVALOS, R. V.; LÓPEZ, O. C. Uma abordagem da implantação de um ERP visando apoio às atividades administrativas e de ensino. In: $3^{\text {a }}$ CONFERÊNCIA DA ASSOCIAÇÃO PORTUGUESA DE SISTEMAS DE INFORMAÇÃO, Coimbra - Portugal. Anais... Coimbra: CAPSI, 2002.

DÁVALOS, R. V.; PLATT, A. A. Implantação de um Sistema Integrado de Gestão visando apoio às atividades universitárias. In: XXVII Congresso Brasileiro de Ensino e Engenharia - COBENGE 2002, Piracicaba - SP.

Anais... Piracicaba: COBENGE, 2002.

FIGUEIREDO, K.; ARKADER, R. Da distribuição física ao supply chain management. agosto de 1998. Disponível em: <http://www.coppead.ufrj.br/ pesquisa/cel.htm>. Acesso em: 21 jun. 2006.

GUTHRIE, R. W.; GUTHRIE, R..A. "Integration of Enterprise System Software in the Undergraduate Curriculum". Proceedings of ISECON, Philadelphia, v. 17, n. 301, 2000.

HAWKING, P.; MCCARTHY, B. The ERP eLearning Model for the Delivery of ERP (SAP R/3) Curriculum into the Asian Region. Informing Science: Challenges to Informing Clients: A Transdisciplinary Approach; June 2001.

HAWKING, P.; FOSTER ,S.; BASSETT, P. An Applied Approach to Teaching HR Concepts Using an ERP System. Informing Science InSITE "Where Parallels Intersect" June 2002.

KURIHARA, T.; BRETERNITZ, V. J. "As ferramentas Enterprise Resource Planning (ERP) e seu impacto nos currículos universitários". Mackenzie de Engenharia e Computação. São Paulo, Ano 1, n. 1, p. 21-29, 2001.

MEDINA, Jovane M. Cadeia de abastecimento no Comércio Eletrônico sob a ótica de redes flexíveis - um método de estruturação. Florianópolis, 2002, 289f. Tese (Doutorado em Engenharia de Produção) Programa de Pós-Graduação em Engenharia de Produção, UFSC, 2002.

PLATT, Allan A. ERP: proposta metodológica de implementação para cursos de graduação. Florianópolis, 2004, 186f. Tese (Doutorado em Engenharia de Produção) - Programa de Pós-Graduação em Engenharia de Produção, UFSC, 2004. 
WATSON, E. E.; SCHNEIDER, H. Using Erp Systems In Education.

Communications of AIS (Association of Information System), v. 1, article 9; february 1999.

WIEDER, B. ERP - Software integration at Australian Universities recent developments in integrated business education. Proceedings of CTI Accounting Finance \& Management Conference. Grã-Bretanha, 1999. 